\title{
Bulk viscosity in a neutron star mantle
}

\author{
D. G. Yakovlev ${ }^{1 \star}$, M. E. Gusakov ${ }^{1}$, P. Haensel $^{2}$ \\ 1 Ioffe Institute, Politekhnicheskaya 26, St Petersburg 194021, Russia \\ 2 Copernicus Astronomical Center, Bartycka 18, 00-716 Warsaw, Poland
}

April 1, 2022

\begin{abstract}
We study the bulk viscosity in two (anti-spaghetti and Swiss cheese) phases of nonspherical nuclei in the mantle of an oscillating neutron star near the boundary with the stellar core. The bulk viscosity is produced by non-equilibrium Urca neutrino emission processes. In the mantle, the direct Urca process may be open (Gusakov et al., 2004 ) if neutrons and protons move in a periodic potential created by a lattice of nonspherical nuclei (which allows the nucleons to have large quasi-momenta and satisfy direct Urca momentum-conservation). This bulk viscosity can dominate over that due to the modified Urca process in the outer stellar core and over the shear viscosity. The bulk viscosity depends strongly on temperature, oscillation frequency and nucleon superfluidity. The enhanced bulk viscosity in the mantle can control propagation and damping of neutron star oscillations.
\end{abstract}

Key words: stars: neutron - dense matter - conduction - neutrinos

\section{INTRODUCTION}

It is widely thought (Shapiro \& Teukolsky 1983) that any neutron star contains a massive core of superdense matter surrounded by a thin crust $(\sim 1$ percent by mass and $\sim 10$ percent by radius) of matter of subnuclear density. The core is supposed to be liquid and contain neutrons (n), protons (p), electrons (e), muons $(\mu)$, and possibly other particles and elementary excitations like hyperons, $\Delta$ isobars, deconfined quarks. The crust is composed of electrons and atomic nuclei as well as of free neutrons (at densities $\rho$ exceeding the neutron drip density $\left.\rho_{\text {drip }} \sim(4-6) \times 10^{11} \mathrm{~g} \mathrm{~cm}^{-3}\right)$. In the bulk of the crust the electrons are strongly degenerate and relativistic, the nuclei are nearly spherical and neutron rich, and the free neutrons are strongly degenerate and possibly superfluid. The stellar core also consists of highly degenerate and strongly interacting fermions (like $\mathrm{n}$ and $\mathrm{p}$ ) which can be superfluid.

The core-crust interface is placed at $\rho_{\mathrm{cc}} \approx \rho_{0} / 2$ (e.g., Haensel et al. 2007) which corresponds to the baryon number densities $n_{\mathrm{b}}=n_{\mathrm{cc}} \approx 0.5 n_{0}$, where $\rho_{0} \approx 2.8 \times 10^{14}$ $\mathrm{g} \mathrm{cm}^{-3}$ and $n_{0}=0.16 \mathrm{fm}^{-3}$ are, respectively, the density and the baryon number density of symmetric nuclear matter at saturation. It is important that many thermodynamic, kinetic and neutrino-emission properties of the crust and core are drastically different. Accordingly, the crust-core interface is very significant for modeling of many processes in

* E-mail: yak@astro.ioffe.ru neutron stars such as magnetic field and thermal evolution, pulsar glitches, stellar oscillations.

An additional complication is introduced by a possible existence of a special nuclear pasta layer (called also nuclear mantle and viewed often as the bottom layer of the crust) in the density range from $\rho \sim 10^{14} \mathrm{~g} \mathrm{~cm}^{-3}$ to $\rho_{\mathrm{cc}}$. This layer appears in some theoretical models of neutron star matter but is not realized in other models (e.g., Ravenhall et al. 1983; Pethick \& Ravenhall 1995; Haensel et al. 2007). In the essence, the atomic nuclei in the nuclear pasta form exotic structures, with the sea of free neutrons (and even free protons) between them. The bulk properties of the nuclear pasta layer (e.g., the equation of state, EOS) remain almost the same as in a fluid of neutrons with the admixture of protons and electrons (almost as in the outer liquid core). Accordingly, the nuclear pasta weakly affects hydrostatic structure of the star but can much stronger affect transport and neutrino emission properties of the mantle (e.g. Lorenz et al. 1993; Gusakov et al. 2004; Horowitz \& Berry 2008; Nandi \& Schramm 2018 and references therein). Although the pasta layer occurs in a narrow density interval, its mass is not much smaller than the total mass of the crust.

In this paper, we focus on the bulk viscosity in the nuclear pasta using the results of Haensel et al. (2000) and Gusakov et al. (2004). The bulk viscosity is required for modeling neutron star oscillations or propagation of waves generated in neutron stars during active periods of their evolution. In particular, it is expected to be important at the stages at which neutron stars become powerful sources of gravitational waves (e.g., in rapidly rotating stars suffering 
r-mode instabilities or during neutron star merging in compact binaries; see Alford et al. 2018 and references therein).

\section{NUCLEAR PASTA AND DIRECT URCA PROCESS}

Nuclear pasta in neutron stars is extensively studied using two major approaches.

First, one considers ordered nuclear clusters, which can exist in four exotic phases I-IV, by minimizing the energy density at a given baryon density. The phase I of rods ('spaghetti') appears after the standard phase of spherical nuclei as $\rho \rightarrow \rho_{\mathrm{cc}}$. Nuclear structures become rod-like there; they are crystallized in a two-dimensional lattice immersed in a liquid of free neutrons. One studies neutron and proton density profiles across the rods assuming that their length is infinite. The phase of rods is followed by the phase II of slabs (the 'lasagna' phase). The slabs are filled by nuclear matter and form a one-dimensional lattice, with the neutron liquid between the slabs. One models the nuclear structure across the slabs treating them as infinite. The next phase III is a two-dimensional lattice of infinite rod-like bubbles of neutron liquid (possibly with some amount of free protons) with nuclear structures between them; it is called 'anti-spaghetti.' The final phase IV consists of a crystal of spherical bubbles of free neutrons (and possibly free protons) called 'Swiss cheese'; nuclear structures fill the space between the bubbles.

These structures are studied using compressible liquid drop models or Thomas-Fermi theories (e.g., Williams \& Koonin 1985; Oyamatsu 1993; Lorenz et al. 1993; Watanabe et al. 2000; Watanabe \& Iida 2003; Nakazato et al. 2009; Okamoto et al. 2013; Grill et al. 2014; Sharma et al. 2015, and references therein). Some calculations reproduce all phases I-IV; others reproduce fewer exotic phases or none of them (e.g. Douchin \& Haensel 2001; Grill et al. 2014). Of course, real rods or slabs should have finite sizes; they are possibly arranged in domains. However, the sizes of clusters and structure of domains cannot be obtained from such calculations.

Second, one simulates nuclear pastas using molecular dynamics (e.g., Maruyama et al. 1998; Kido et al. 2000; Watanabe et al. 2002; Schuetrumpf et al. 2015; Horowitz et al. 2004, 2005; Dorso et al. 2012; Caplan et al. 2015; Horowitz et al. 2015 and references therein). These simulations are sensitive to the models of interacting nucleons, but they have more degrees of freedom. Accordingly, they predict nuclear structures of different sizes, shapes and orientations. One obtains a rich variety of pasta models, for instance, nuclear clusters of single type (e.g., slabs) with different parameters for a given density of the matter; mixtures of cluster types (e.g., slabs and rods); even more exotic clusters, called nuclear waffles, gyroids, intertwined lasagna, long lived topological defects and so on (e.g., Schneider et al. 2014; Nakazato et al. 2009; Schuetrumpf et al. 2015; Alcain et al. 2014; Horowitz et al. 2015). At temperatures $T \lesssim 10^{10} \mathrm{~K}$ of our interest, such nuclear pastas usually freeze into ordered or disordered systems.

All these studies give rather diverse results - wealth of possibilities. To be specific, we employ the well-known model
I of Oyamatsu (1993). It is the nuclear pasta model of the first type containing all four basic pasta layers I-IV. The phase I appears at density $\rho_{1}=0.973 \times 10^{14} \mathrm{~g} \mathrm{~cm}^{-3}$ (at baryon number density $n_{1}=0.0586 \mathrm{fm}^{-3}$ ). The phase II occurs at $\rho_{2}=1.24 \times 10^{14} \mathrm{~g} \mathrm{~cm}^{-3}\left(n_{2}=0.0749 \mathrm{fm}^{-3}\right)$, and the phase III at $\rho_{3}=1.37 \times 10^{14} \mathrm{~g} \mathrm{~cm}^{-3}\left(n_{3}=0.0827 \mathrm{fm}^{-3}\right)$. The final phase IV appears at $\rho_{4}=1.42 \times 10^{14} \mathrm{~g} \mathrm{~cm}^{-3}$ $\left(n_{4}=0.0854 \mathrm{fm}^{-3}\right)$ and disappears at the crust-core interface $\left(\rho_{\mathrm{cc}}=1.43 \times 10^{14} \mathrm{~g} \mathrm{~cm}^{-3}, n_{\mathrm{cc}}=0.0861 \mathrm{fm}^{-3}\right)$.

In the phases III and IV, free (unbound) protons appear in a liquid of free neutrons. It happens not only in the models by Oyamatsu (1993) but in some other models (see, e.g., Sharma et al. 2015). It is favorable for opening a powerful direct Urca process of neutrino emission in the neutron star mantle. The idea was put forward by Lorenz et al. (1993) and realized by Gusakov et al. (2004).

It is well known (Lattimer et al. 1991) that the direct Urca process can produce the strongest neutrino emission in the inner cores of neutron stars, at $\rho \gtrsim(2-3) \rho_{0}$. The simplest direct Urca process involves neutrons, protons and electrons; it is a chain of two (direct and inverse) reactions,

$$
\mathrm{n} \rightarrow \mathrm{p}+\mathrm{e}+\bar{\nu}_{\mathrm{e}}, \quad \mathrm{p}+\mathrm{e} \rightarrow \mathrm{n}+\nu_{\mathrm{e}}
$$

where $\nu_{\mathrm{e}}$ and $\bar{\nu}_{\mathrm{e}}$ are the electron neutrino and antineutrino, respectively. In a strongly degenerate matter the momenta of neutrons, protons and electrons should be close to their Fermi momenta $p_{\mathrm{Fn}}, p_{\mathrm{Fp}}$, and $p_{\mathrm{Fe}}$. The process is allowed by momentum conservation if $p_{\mathrm{Fn}}<p_{\mathrm{Fp}}+p_{\mathrm{Fe}}$, and forbidden otherwise. Typical neutrino momenta $p_{\nu} \sim k_{\mathrm{B}} T / c$ are much smaller than these Fermi momenta and can be neglected in momentum conservation. Because of momentum conservation restriction, the direct Urca process in the neutron star core is allowed only at rather high densities (typically, a few times of $\rho_{0}$ ) for those model EOSs of dense matter which have a large symmetry energy (rather high fractions of protons and electrons) to satisfy the triangle inequality $p_{\mathrm{Fn}}<p_{\mathrm{Fp}}+p_{\mathrm{Fe}}$. In the outer core of the star, this process is forbidden, and only weaker processes like the modified Urca one can operate (e.g. Yakovlev et al. 2001), with the direct and inverse reactions $\mathrm{nN} \rightarrow \mathrm{peN} \bar{\nu}_{\mathrm{e}}$ and $\mathrm{peN} \rightarrow \mathrm{nN} \nu_{\mathrm{e}}, \mathrm{N}$ being a nucleon.

However, crystalline lattices of nuclear structures in the phases III and VI of the neutron star mantle modulate motion of free neutrons and protons (inducing Bloch states and relaxing momentum conservation due to Bragg diffraction). This can open the direct Urca process owing to Umklapp transitions of $\mathrm{n}$ and $\mathrm{p}$. The Umklapp transitions make the direct Urca process in the mantle weaker than in the inner core but it can still be stronger than the modified Urca processes in the outer core of neutron stars. Gusakov et al. (2004) calculated the neutrino emissivity of the direct Urca processes in the phases III and IV of the nuclear pasta using two models for evaluating the matrix elements of the processes. The results appeared to be quite similar and were fitted by an analytic expression. They will be used for calculating the bulk viscosity.

\section{BULK VISCOSITY}

Let us consider the bulk viscosity in the stellar mantle and compare it with the bulk viscosity in the outer core. We will 
mainly neglect the effects of neutron and proton superfluidities but comment on these effects whenever necessary.

\subsection{Stellar core}

The bulk viscosity $\zeta$ has been studied attentively for the conditions in neutron star cores. Its nature is very different from that for the standard shear viscosity $\eta$ determined by frequent collisions between the particles in dense matter (Flowers \& Itoh 1979).

A noticeable bulk viscosity in neutron star cores is produced by slow irreversible beta processes. For simplicity, we consider a matter of neutrons, protons and electrons in an outer core of the star near the crust-core interface. Let us assume that the matter is initially in full thermodynamic equilibrium, including beta-equilibrium. Then the imbalance of the chemical potentials $\delta \mu=\mu_{\mathrm{n}}-\mu_{\mathrm{p}}-\mu_{\mathrm{e}}$ is zero. At the next step we consider weak vibrations of the star with a local oscillation frequency $\omega$. They can be produced by global oscillations of the star or by a propagation of some sound waves. Frequent collisions of the particles will support local thermodynamic equilibrium but Urca processes are too slow to restore beta equilibrium during oscillations. Oscillations of particle number densities and the pressure $P$ will be slightly shifted in phase, producing dissipation that can be described by an effective bulk viscosity $\zeta$.

The first calculations of $\zeta$ have been conducted by Sawyer (1989); Haensel \& Schaeffer (1992); we will follow the consideration by Haensel et al. (2000, 2001). The bulk viscosity is controlled by non-equilibrium (direct and/or modified) Urca processes and depends on the oscillation frequency $\omega$. For simplicity, we restrict ourselves by the so called linear regime, where the amplitude $|\delta \mu|$ of $\delta \mu$ oscillations is smaller than $k_{\mathrm{B}} T, k_{\mathrm{B}}$ being the Boltzmann constant. Note that neutron star oscillations in the non-linear regime $\left(|\delta \mu| \gg k_{\mathrm{B}} T\right)$ were studied e.g., by Finzi \& Wolf (1968); Gusakov et al. (2005); Alford et al. (2012). In the linear regime, the difference of the rates $\Delta \Gamma$ of the direct and inverse Urca reactions is linear in $\delta \mu$,

$$
\Delta \Gamma=-\lambda \delta \mu,
$$

where $\lambda$ is a factor that can be expressed through the rate $\Gamma_{0}$ of equilibrium Urca processes. For the direct Urca process (Haensel et al. 2000),

$$
|\lambda|=\frac{17 \pi^{4}}{60} \frac{\Gamma_{0}}{k_{\mathrm{B}} T}
$$

with $\Gamma_{0}$ given by Eq. (28) of Haensel et al. (2000). In this case $\lambda \propto T^{4}$. The expressions for $\lambda$ mediated by the modified Urca processes are given in Haensel et al. (2001), with $\lambda \propto$ $T^{6}$. If neutrons and/or protons are superfluid, neutrino reaction rates are reduced due to the appearance of gaps in the energy spectra of nucleons (e.g. Yakovlev et al. 2001). Then the contribution of any Urca process has to be multiplied by a corresponding reduction factor (e.g., Haensel et al. 2000, 2001). The reduction factors can strongly (exponentially) depend on $T$, violating simple power-law temperature dependence of $\lambda$. If several Urca processes operate at once, one should sum corresponding reaction factors $\lambda$. Since $\lambda$ is sometimes introduced with different signs we use $|\lambda|$ to avoid misunderstandings.
In the npe-matter, the effective bulk viscosity can be written as (Haensel et al. 2000)

$$
\zeta=\frac{C_{\mathrm{e}}^{2} n_{\mathrm{b}}^{2}|\lambda|}{B_{\mathrm{ee}}^{2}|\lambda|^{2}+\omega^{2} n_{\mathrm{b}}^{2}},
$$

where $n_{\mathrm{b}}$ is the baryon number density at equilibrium, while

$$
C_{\mathrm{e}}=-\frac{1}{n_{\mathrm{b}}} \frac{\partial P\left(n_{\mathrm{b}}, X_{\mathrm{e}}\right)}{\partial X_{\mathrm{e}}}, \quad B_{\mathrm{ee}}=n_{\mathrm{b}} \frac{\partial \delta \mu\left(n_{\mathrm{b}}, X_{\mathrm{e}}\right)}{\partial n_{\mathrm{b}}},
$$

are the derivatives of thermodynamic pressure and chemical potential imbalance with respect to $X_{\mathrm{e}}$ (the ratio of the numbers of electrons and baryons) and $n_{\mathrm{b}}$. The derivatives have to be calculated by considering $n_{\mathrm{b}}$ and $X_{\mathrm{e}}$ as independent variables and taken at equilibrium. To a good approximation, they are independent of $T$.

For expected vibration frequencies $\omega \sim 10^{3}-10^{4} \mathrm{~s}^{-1}$ the high-frequency limit of (4) is an excellent approximation,

$$
\zeta=\frac{C_{\mathrm{e}}^{2}|\lambda|}{\omega^{2}} .
$$

This bulk viscosity decreases as $\omega^{-2}$ with growing $\omega$. In a non-superfluid matter, it increases with the growth of $T$.

In the unrealistic low-frequency limit $(\omega \rightarrow 0)$ the bulk viscosity becomes static and huge,

$$
\zeta=\frac{C_{\mathrm{e}}^{2} n_{\mathrm{b}}^{2}}{B_{\mathrm{ee}}^{2}|\lambda|}
$$

Its dependence on $|\lambda|$ is inverted, inverting the temperature dependence of $\zeta$.

A transition from the high-frequency to the lowfrequency regime occurs at $\omega=\omega_{0} \sim\left|B_{\text {ee }} \lambda\right| / n_{\mathrm{b}}$. Some estimates of $\omega_{0}$ are given in Section 3.3. The estimates of $\lambda$ and $C_{\mathrm{e}}$ can be found in Haensel et al. $(2000,2001) ; B_{\text {ee }}$ is negative, with $\left|B_{\text {ee }}\right| \sim$ a few $\mathrm{GeV}$.

\subsection{Pasta layers}

The consideration of Section 3.1 implies that the bulk viscosity in nuclear pasta is given by the same Eq. (4) with the corresponding value of $\lambda$. A comparison of the results of Haensel et al. (2000) and Gusakov et al. (2004) indicates that the factor $\lambda$ in the pasta layers III and IV is given by the same expression (3) corrected for the reduction of the direct Urca process due to the presence of nuclear structures,

$$
|\lambda|=\frac{17 \pi^{4}}{60} \frac{\Gamma_{0}}{k_{\mathrm{B}} T} \mathcal{R}
$$

Here $\mathcal{R}=\mathcal{R}\left(n_{\mathrm{b}}\right)$ is the reduction factor given by Eq. (12) of Gusakov et al. (2004) who approximated it at $n_{3} \leqslant n_{\mathrm{b}} \leqslant n_{\mathrm{cc}}$ by their Eq. (15),

$$
\begin{aligned}
& \mathcal{R}\left(n_{\mathrm{b}}\right)=\mathcal{R}_{2}+\left(\mathcal{R}_{1}-\mathcal{R}_{2}\right)(1-x)^{2} \quad x<1, \\
& \mathcal{R}\left(n_{\mathrm{b}}\right)=\mathcal{R}_{2} / x^{7} \quad x \geqslant 1,
\end{aligned}
$$

where $\mathcal{R}_{1}=6 \times 10^{-5}, \mathcal{R}_{2}=10^{-5}$, and $x=\left(n_{\mathrm{b}}-\right.$ $\left.n_{3}\right) /\left(n_{4}-n_{3}\right)$, with $n_{3}=0.0827 \mathrm{fm}^{-3}, n_{4}=0.0854 \mathrm{fm}^{-3}$ and $n_{\mathrm{cc}}=0.0861 \mathrm{fm}^{-3}$. If free nucleons in the pasta are superfluid, Eq. (8) should be multiplied by the additional superfluid reduction factor, which is the same as discussed by Gusakov et al. (2004). Equations (4), (8) and (9) allow one to evaluate $\zeta$ in the pasta layers. 


\subsection{Illustrative examples}

Fig. 1 illustrates the behavior of the bulk viscosity $\zeta$ (solid lines) as a function of $n_{\mathrm{b}}$ in the pasta layers III and IV and in the outer layer of the core (neglecting the effects of superfluidity). The left-hand panel is for the temperature $T=10^{9}$ $\mathrm{K}$, while the right-hand panel is for $T=10^{8} \mathrm{~K}$. The pasta phase III occurs between the left and middle dotted vertical lines while the phase IV is realized between the middle and right dotted vertical lines. Higher $n_{\mathrm{b}}$ correspond to the stellar core. Different solid lines are plotted for different oscillation frequencies $\omega$; the upper solid line shows the static $\zeta$ at $\omega \rightarrow 0$.

In the core, we use a simple model EOS of npe $\mu$-matter suggested by Heiselberg \& Hjorth-Jensen (1999). Specifically, we use the same version of this EOS as employed by Kaminker et al. (2014) who called it $\operatorname{HHJ}(0.1,0.7)$. Its advantage is that one can easily calculate the thermodynamic derivatives (5). For this EOS, the maximum gravitational mass of stable neutron stars is $M_{\max }=2.16 \mathrm{M} \odot$. The central density of the maximum-mass star is $\rho=2.45 \times 10^{15}$ $\mathrm{g} \mathrm{cm}^{-3}\left(n_{\mathrm{b}}=1.06 \mathrm{fm}^{-3}\right)$, and the circumferential radius $R=10.84 \mathrm{~km}$. The direct Urca processes operate in stars with $M>1.77 \mathrm{M} \odot$ (with central densities $>1.05 \times 10^{15}$ $\mathrm{g} \mathrm{cm}^{-3}$ and $\left.n_{\mathrm{b}}>0.558 \mathrm{fm}^{-3}\right)$. The muons appear in the core at $\rho=2.32 \times 10^{14} \mathrm{~g} \mathrm{~cm}^{-3}\left(n_{\mathrm{b}}=0.137 \mathrm{fm}^{-3}\right)$, just near the outer core boundary. Their appearance induces the modified Urca process with muons and slightly increases $\zeta$. The nuclear pasta model in Fig. 1 is the same model by Oyamatsu (1993) as described in Section 2. The bulk viscosity we discuss operates in the pases III and IV.

Thermodynamic derivatives (5) in the core are calculated in the same manner as in Haensel et al. (2000, 2001). The bulk viscosity in the outer npe-layer of the core is computed using Eq. (4). For deeper layers, containing muons, we have used Eq. (9) of Haensel et al. (2000).

The thermodynamic derivatives (5) in the pasta layers are, in principle, affected by nuclear structures. We have tried to include this effect by using the so called bulk approximation described, e.g. in Haensel et al. (2007), Section 3.4.1. However, because the pasta layers III and IV are very close to the core, the effect is not large, and, for simplicity, we determine the required derivatives in the same way as in the outer npe-core. Note that the factor $\mathcal{R}$ in (9) is obtained (Gusakov et al. 2004) for the model of Oyamatsu (1993). This means that the curves in Fig. 1 are plotted for a mixture of EOSs of dense matter. It is a typical situation in neutron star physics, that different layers of the star are described by different EOSs. Regretfully, we have no data to calculate $\zeta$ in the core using the results by Oyamatsu (1993). Equally, we cannot accurately calculate $\zeta$ in the mantle using the HHJ EOS because we have no data on nuclear pasta for that EOS. Nevertheless, as always happens in such cases, we can expect that Fig. 1 is qualitatively correct.

For comparison, by long-dashed lines in Fig. 1 we present also the shear viscosities $\eta$ in the innermost layer of the crust and in the outermost core layer. In the core, we plot $\eta$ using the same HHJ EOS and results of Shternin \& Yakovlev (2008). In the crust, we employ the results by Chugunov \& Yakovlev (2005); Shternin (2008). In the latter case we use the smooth composition model of the inner crust (Haensel et al. 2007, Appendix B.2.1) neglect- ing the presence of nuclear pasta. Note that the shear viscosity in the pasta layers was studied by Horowitz \& Berry (2008); Nandi \& Schramm (2018), who showed that it does not differ drastically from $\eta$ calculated for spherical nuclei. In contrast to $\zeta$, the shear viscosity is independent of $\omega$.

As seen from Fig. 1, the bulk viscosity is very sensitive to the temperature and oscillation frequency $\omega$. The expected oscillation frequencies are $\omega \sim 10^{3}-10^{4} \mathrm{~s}^{-1}$, but we consider a much wider range of $\omega$ for the completeness of our study. Typically, the high-frequency regime (6) is seen to be an excellent approximation, so that $\zeta \propto T^{4} / \omega^{2}$ in the pasta and $\zeta \propto T^{6} / \omega^{2}$ in the outer core (Sections 3.1 and 3.2). Accordingly, $\zeta$ decreases with decreasing $T$ (e.g. during neutron star cooling) and increasing $\omega$. The bulk viscosity is especially effective in warm neutron stars, where Urca processes are stronger. In any case the bulk viscosity in the outer core (powered by the modified Urca process) is smaller than in the pasta, where it is powered by the direct Urca process partially suppressed by the crystalline structure effects (Gusakov et al. 2004). In the inner cores of neutron stars the direct Urca processes are stronger and $\zeta$ can be much larger. This is not shown in Figure 1 but is well known (e.g., Haensel et al. 2000, 2001).

According to Fig. 1, at any temperature and density there exists some minimum oscillation frequency $\omega_{*}$ above which the bulk viscosity $\zeta$ becomes smaller than the shear viscosity $\eta$. For instance, at $T=10^{9} \mathrm{~K}$ in the pasta $\omega_{*} \sim 10^{6}$ $\mathrm{s}^{-1}$, while in the outer core $\omega_{*} \sim 3 \times 10^{4} \mathrm{~s}^{-1}$. At $T=10^{8}$ $\mathrm{K}$ we have $\omega_{*} \sim 10^{2} \mathrm{~s}^{-1}$ in the pasta and $\omega_{*} \sim 3 \mathrm{~s}^{-1}$ in the outer core.

Let us recall that we use one $\operatorname{EOS}, \operatorname{HHJ}(0.1,0.7)$, in the neutron star core. A comparison with the results by Haensel et al. (2000, 2001), who used two other, sufficiently different EOSs, reveals that at the same $\omega$ and $T$ the values of $\zeta$ for different EOSs are close (differ by a factor of few). The largest difference occurs at densities of muon appearance and at threshold densities for opening Urca processes. It seems that by varying the EOS in the non-superfluid core one cannot noticeably change the values of $\zeta$. The same is so for the values of $\eta$ and $\omega_{*}$.

With decreasing $\omega$, the transition to the static $\zeta$, Eq. (7), takes takes place at unrealistically low frequencies, $\omega_{0} \sim$ $10^{-6} \mathrm{~s}^{-1}$ for $T=10^{9} \mathrm{~K}$ and $\omega_{0} \sim 10^{-12} \mathrm{~s}^{-1}$ for $T=10^{8} \mathrm{~K}$. As already mentioned above, the static $\zeta$ has the inverted temperature dependence with respect to the high-frequency $\zeta$. The static $\zeta$ in the pasta becomes lower than in the outer core, and the static $\zeta$ increases with decreasing $T$.

If nucleons are superfluid, one should calculate $\zeta$ by introducing superfluid reduction factors in the quantities $\lambda$ as discussed above. For instance, in the high-frequency limit superfluidity always suppresses $\zeta$ in exactly the same manner as discussed by Haensel et al. $(2000,2001)$ for the case of stellar core. The suppression of $\zeta$ in the pasta is the same as the reduction of corresponding neutrino emissivity of the direct Urca process analyzed by Gusakov et al. (2004).

Therefore, in contrast to the effects of EOS, which affect $\zeta$ rather slightly, the effects of superfluidutity of neutrons and protons are really dramatic. The most important parameters there are critical temperatures of neutrons and protons as functions of density (e.g. Page et al. 2014 and references therein). They are extremely model-dependent, very sensitive to in-medium effects in dense matter. The region of the 

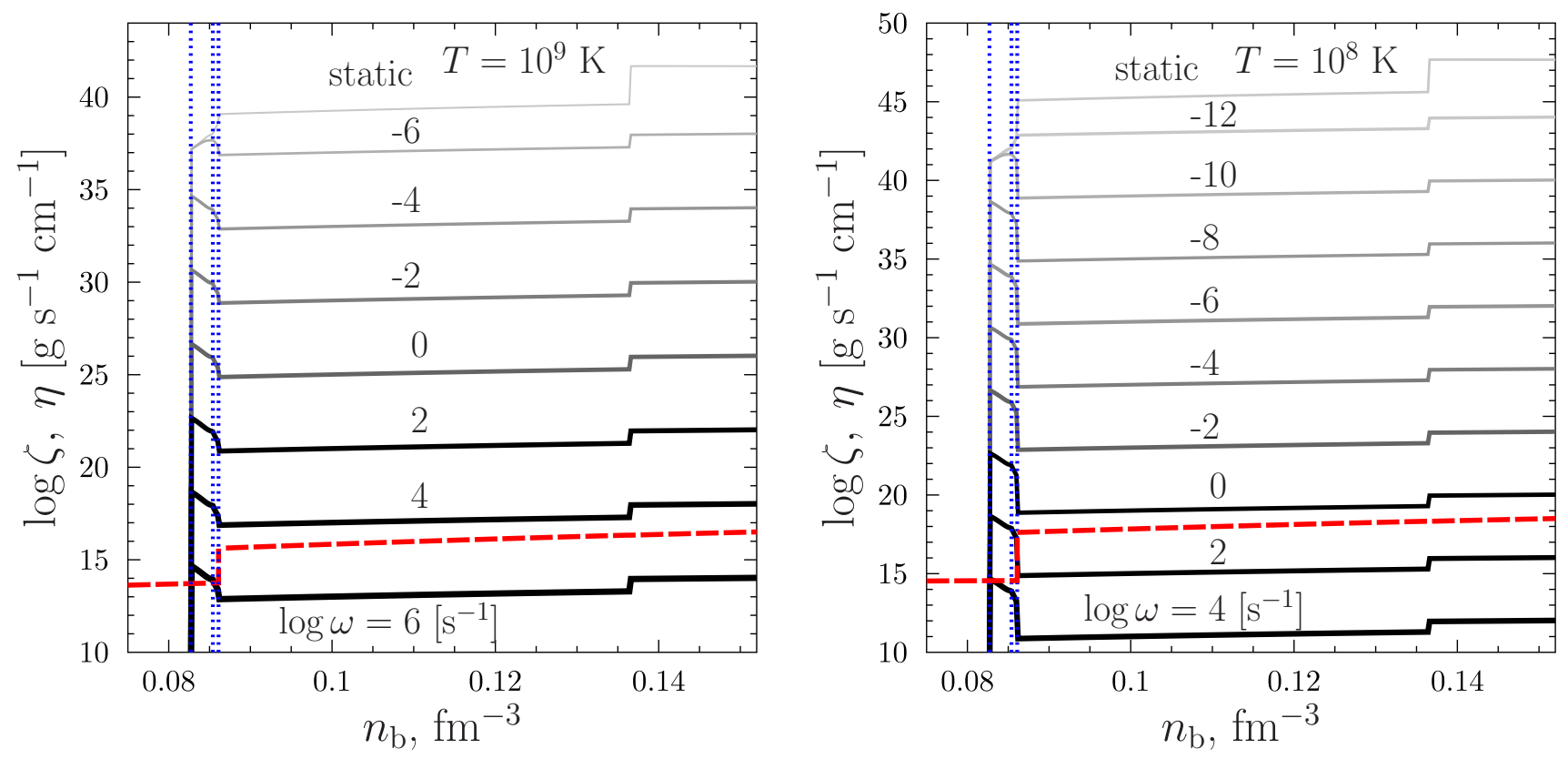

Figure 1. (Color online) Bulk viscosity $\zeta$ (solid lines) versus baryon number density $n_{\mathrm{b}}$ in the pasta layers III and IV and in the outer layer of the core at $T=10^{9} \mathrm{~K}$ (left) and $10^{8} \mathrm{~K}$ (right). Lines of different thickness and grayness refer to different vibration frequencies $\omega$ (the values of $\log \omega$ are given near the curves); the upper light gray lines show the static $\zeta$ (at $\omega=0)$. For comparison, the long-dashed lines demonstrate the shear viscosity $\eta$. The left dotted vertical line in each panel shows the outer boundary of the anti-spaghetti pasta layer III; the next vertical line positions the onset of the Swiss-cheese layer IV, and the right vertical line marks the outer boundary of the stellar core. The effects of superfluidity are neglected. See text for details.

crust-core interface is just the place where theoretical models for superfluidity are very divergent. If superfluidity in the mantle is strong it can completely suppress high-frequency $\zeta$ there (as follows from the results by Gusakov et al. 2004). If superfluidity is stronger in the core, it will suppress the bulk viscosity in the core (Haensel et al. 2001). Another important effect of superfluidity on the bulk viscosity is described in the next section.

\section{DISCUSSION}

The effective bulk viscosity considered above is well known in hydrodynamics of liquids in which slow chemical reactions are possible (e.g., Landau \& Lifshitz 1987). It produces viscous heat to be included in the equation of heat transport and effective viscous force in the Navier-Stokes equation. In vibrating neutron stars, such a bulk viscosity appears because vibrations violate beta equilibrium and induce slow (weak) non-equilibrium processes which drive the system to the full equilibrium. Such processes are not necessarily associated with the emission of neutrinos but can be governed, for instance, by weak reactions between hyperons (e.g., Gusakov \& Kantor 2008).

Let us stress that there is also a standard bulk viscosity mediated by frequent interparticle collisions (e.g. Lifshitz \& Pitaevskiĭ 1981). It is quite similar to traditional transport coefficients like the shear viscosity or thermal conductivity. In particular, it is independent of oscillation frequency and can be used for any (not only pulsating) motions. However, under the neutron star conditions this 'standard' bulk viscosity seems mainly negligible. The frequency de- pendence in our case occurs because non-equilibrium beta processes can be much slower than oscillations.

The calculated bulk viscosity is valid under restricted conditions. First of all, the variations of the chemical potential imbalance, by construction (e.g. Haensel et al. 2000), have to be small, $|\delta \mu| \ll k_{\mathrm{B}} T$. Secondly, such a bulk viscosity is obtained by averaging over one pulsation cycle (see, again, Haensel et al. 2000). Note that in the static limit $(\omega \rightarrow 0)$ the latter approximation can be violated, and the problem should be considered more carefully. However, under the neutron star conditions, this limit is unrealistic. Accordingly, we use the available expressions at $\omega \rightarrow 0$ just to show the variation amplitude of bulk viscosity values. Thirdly, for the presented expressions to be valid, the matter should oscillate as a whole, whereas sometimes several matter components oscillate differently. This is especially so if neutrons and/or protons are superfluid; such superfluid components of the matter may move separately which naturally leads to several coefficients of bulk viscosity (e.g., Gusakov 2007; Gusakov \& Kantor 2008). Finally, matter motions are not necessarily reduced to pure oscillations at a fixed frequency $\omega$. The motions can be complicated and the velocity can be an arbitrary function of time $t$, irreducible to oscillations with fixed $\omega$.

In all these cases, the relaxation of stellar perturbations due to non-equilibrium beta processes can be taken into account by introducing a system of hydrodynamic equations for different components of the matter (see, e.g., Gusakov et al. 2005; Kantor \& Gusakov 2009 for an example of such calculations). Various quantities in these equations (for instance, chemical potentials) can be out of equilibrium and have to be treated as functions of a local baryon 
number density $n_{\mathrm{b}}$ and local instantaneous particle fractions. A solution of such a system automatically includes all the effects described by the phenomenon of bulk viscosity. This approach can also be valid in the non-linear regime in which $|\delta \mu| \gg k_{\mathrm{B}} T$ and beta-equilibration is greatly accelerated by strong violations from beta-equilibrium.

Let us recall that the bulk and shear viscosities damp different types of motions $\boldsymbol{v}(\boldsymbol{r}, t)$. The shear viscosity affects shear motions while the bulk viscosity influences compression-rarefaction motions with $\operatorname{div} \boldsymbol{v} \neq 0$. For instance, standard sound waves are damped by both viscosities (e.g., Landau \& Lifshitz 1987). In a matter with a not too strong magnetic fields $\boldsymbol{B}$, where $B^{2} / 8 \pi \ll P$, the fast magnetic sound waves are also damped as the ordinary sound, but the Alfvénic waves and slow magnetic sound waves have shear-like structure and they are mostly damped by the shear viscosity. The damping of proper oscillations of the star is determined by configurations of fluid motion in these oscillations.

\section{CONCLUSIONS}

We have calculated the bulk viscosity in the two (antispaghetti and Swiss cheese) phases of nuclear pasta in the mantle of vibrating neutron stars. The pasta phases are described by model 1 of Oyamatsu (1993). The bulk viscosity is mediated by direct Urca process which is opened (Gusakov et al. 2004) because free neutrons and protons are moving there in a periodic potential created by a lattice of nuclear structures.

We have compared the calculated bulk viscosity in the pasta with the bulk viscosity in the outer neutron star core and with appropriate shear viscosities. The bulk viscosity appears to be a strong function of temperature, density and vibration frequency $\omega$. For typical vibration frequencies $\omega \sim$ $10^{3}-10^{4} \mathrm{~s}^{-1}$ the bulk viscosity in the pasta exceeds the bulk viscosity in the outer core as well as the shear viscosity in the core and in the crust at high enough temperatures $T \gtrsim(1.5-$ $3) \times 10^{8} \mathrm{~K}$. In addition, the bulk viscosity can be drastically affected (mostly suppressed) by superfluidity (Section 3.3). The bulk viscosity can influence the propagation of waves through the pasta layer and damping of various neutron star oscillations, as well as of various hydrodynamical motions in the vicinity of the pasta layer. It can also affect damping of perturbations in a neutron star merging with a compact companion during the emission of gravitational waves (e.g., Alford et al. 2018; Alford \& Harris 2018).

It is important to stress that the employed pasta model (Oyamatsu 1993) is just one example in a sea of many diverting models (Section 2). Particularly, that model essentially requires pure crystalline lattice of exotic nuclear structures. Even a slight lattice disorder can affect the bulk viscosity via the factor $\mathcal{R}$ in Eq. (9) (through summations over lattice vectors in Eqs. (8) and (12) of Gusakov et al. 2004; note also an approximate character of these summations which would be desirable to elaborate in the future). Even for pure lattice, the bulk viscosity can be drastically changed by superfluidity. The bulk viscosities for other pasta models have not been studied in the literature. Different pasta models should provide a wealth of speculations regarding transport and neutrino emission properties of the pasta layers (a huge job for future studies).

For the bulk viscosity (and the direct Urca process) in the mantle in our model to be strong, one needs the presence of free neutrons and protons. Note that some models of neutron star matter predict the appearance of free protons in a neutron star crust (near the core) even in the absence of nuclear pasta (e.g., Potekhin et al. 2013). This may trigger additional neutrino emission and associated bulk viscosity at the bottom of the ordinary crust.

As already mentioned above, molecular dynamics simulations of electron thermal and electrical conductivities and shear viscosity in the pasta layers (e.g. Horowitz \& Berry 2008; Nandi \& Schramm 2018) indicate that kinetic properties of nuclear pasta are not essentially different from those in the inner crust of neutron stars with spherical nuclei. This would mean that the pasta layer is not a special layer possessing extraordinary kinetic properties.

We note that the above conclusion is based on molecular dynamics models of pasta layers (Section 2), whereas we use the pasta model of other type whose kinetic properties have not been thoroughly investigated; for our pasta model, the conclusion might be different. In any case, the situation with the bulk viscosity can be different because the bulk viscosity is too different from other transport coefficients. The bulk viscosity seems to be much more sensitive to the properties of pasta layers and it can play a special role there. It is strongly affected by superfluidity. In addition, the conclusion on the standard kinetic properties of the pasta layers, obtained from molecular dynamics, has been made neglecting the effects of strong magnetic fields, while the presence of such fields can drastically affect all the kinetic properties of the pasta layers that is almost unexplored (Yakovlev 2015). It might be so that the magnetic fields make kinetic properties of nuclear pasta extraordinary. In particular, the electron shear viscosity in a magnetic field becomes much more complicated (e.g., Ofengeim \& Yakovlev 2015) than the thermal or electrical conductivities.

Finally, in less deep crustal layers the problem of beta equilibrium is also important. In the essence, beta equilibrium is mediated by beta processes on highly unusual, neutron rich nuclei (direct and inverse reactions of Urca processes on nuclei). Such processes can be extremely slow and keep the crust out of beta equilibrium for a very long time except for those places in the crust where Urca pairs of atomic nuclei coexist (e.g. Imshennik et al. 1968; Beaudet et al. 1972; Deibel et al. 2016, and references therein) which could strongly accelerate beta-equilibration.

In summary, there are many properties of neutron star crust and mantle, which need to be explored that is definitely out of the scope of the present paper.

\section{ACKNOWLEDGMENTS}

The work of PH was supported by the Polish National Science Centre (NCN) (grant no. 2013/11/B/ST9/04528), the work of MG by the Foundation for the Advancement of Theoretical Physics BASIS [Grant No. 17-12-204-1 (MEG)], and the work of DY was partly supported by the Russian Foundation for Basic Research (grant 16-29-13009-ofi-m). 


\section{References}

Alcain P. N., Giménez Molinelli P. A., Dorso C. O., 2014, Phys. Rev. C, 90, 065803

Alford M. G., Harris S. P., 2018, preprint (arXiv:1803.00662)

Alford M. G., Mahmoodifar S., Schwenzer K., 2012, Phys. Rev. $\mathrm{D}, 85,044051$

Alford M. G., Bovard L., Hanauske M., Rezzolla L., Schwenzer K., 2018, Physical Review Letters, 120, 041101

Beaudet G., Salpeter E. E., Silvestro M. L., 1972, Astrophys. J., 174,79

Caplan M. E., Schneider A. S., Horowitz C. J., Berry D. K., 2015, Phys. Rev. C, 91, 065802

Chugunov A. I., Yakovlev D. G., 2005, Astronomy Reports, 49, 724

Deibel A., Meisel Z., Schatz H., Brown E. F., Cumming A., 2016, Astrophys. J., 831, 13

Dorso C. O., Giménez Molinelli P. A., López J. A., 2012, Phys. Rev. C, 86, 055805

Douchin F., Haensel P., 2001, Astron. Astrophys., 380, 151

Finzi A., Wolf R. A., 1968, ApJ, 153, 835

Flowers E., Itoh N., 1979, Astrophys. J., 230, 847

Grill F., Pais H., Providência C., Vidaña I., Avancini S. S., 2014, Phys. Rev. C, 90, 045803

Gusakov M. E., 2007, Phys. Rev. D, 76, 083001

Gusakov M. E., Kantor E. M., 2008, Physical Review D, 78, 083006

Gusakov M. E., Yakovlev D. G., Haensel P., Gnedin O. Y., 2004, Astron. Astrophys., 421, 1143

Gusakov M. E., Yakovlev D. G., Gnedin O. Y., 2005, MNRAS, 361,1415

Haensel P., Schaeffer R., 1992, Physical Review D, 45, 4708

Haensel P., Levenfish K. P., Yakovlev D. G., 2000, Astron. Astrophys., 357, 1157

Haensel P., Levenfish K. P., Yakovlev D. G., 2001, Astron. Astrophys., 372, 130

Haensel P., Potekhin A. Y., Yakovlev D. G., 2007, Neutron Stars. 1. Equation of State and Structure. Springer, New York

Heiselberg H., Hjorth-Jensen M., 1999, Astrophys. J. Lett., 525, L45

Horowitz C. J., Berry D. K., 2008, Phys. Rev. C, 78, 035806

Horowitz C. J., Pérez-García M. A., Carriere J., Berry D. K., Piekarewicz J., 2004, Phys. Rev. C, 70, 065806

Horowitz C. J., Pérez-García M. A., Berry D. K., Piekarewicz J., 2005, Physical Review C, 72, 035801

Horowitz C. J., Berry D. K., Briggs C. M., Caplan M. E., Cumming A., Schneider A. S., 2015, Physical Review Letters, 114, 031102

Imshennik V. S., Nadezhin D. K., Pinaev V. S., 1968, Sov. Astron., 11, 617

Kaminker A. D., Kaurov A. A., Potekhin A. Y., Yakovlev D. G., 2014, MNRAS, 442, 3484

Kantor E. M., Gusakov M. E., 2009, Phys. Rev. D, 79, 043004

Kido T., Maruyama T., Niita K., Chiba S., 2000, Nuclear Physics A, 663,877

Landau L. D., Lifshitz E. M., 1987, Fluid Mechanics. Pergamon, Oxford

Lattimer J. M., Pethick C. J., Prakash M., Haensel P., 1991, Physical Review Letters, 66, 2701

Lifshitz L. M., Pitaevskiı̌ L. P., 1981, Physical Kinetics. Butterworth-Heinemann, Oxford

Lorenz C. P., Ravenhall D. G., Pethick C. J., 1993, Physical Review Letters, 70,379

Maruyama T., Niita K., Oyamatsu K., Maruyama T., Chiba S., Iwamoto A., 1998, Phys. Rev. C, 57, 655

Nakazato K., Oyamatsu K., Yamada S., 2009, Physical Review Letters, 103, 132501

Nandi R., Schramm S., 2018, ApJ, 852, 135
Ofengeim D. D., Yakovlev D. G., 2015, EPL (Europhysics Letters), 112, 59001

Okamoto M., Maruyama T., Yabana K., Tatsumi T., 2013, Phys. Rev. C, 88, 025801

Oyamatsu K., 1993, Nuclear Physics A, 561, 431

Page D., Lattimer J. M., Prakash M., Steiner A. W., 2014, in Bennemann K. H., Ketterson J. B., eds, Novel Superfluids. Oxford Univ. Press, Oxford, p. 550

Pethick C. J., Ravenhall D. G., 1995, Annual Review of Nuclear and Particle Science, 45, 429

Potekhin A. Y., Fantina A. F., Chamel N., Pearson J. M., Goriely S., 2013, Astron. Astrophys., 560, A48

Ravenhall D. G., Pethick C. J., Wilson J. R., 1983, Physical Review Letters, 50, 2066

Sawyer R. F., 1989, Phys. Rev. D, 39, 3804

Schneider A. S., Berry D. K., Briggs C. M., Caplan M. E., Horowitz C. J., 2014, Phys. Rev. C, 90, 055805

Schuetrumpf B., Klatt M. A., Iida K., Schröder-Turk G. E., Maruhn J. A., Mecke K., Reinhard P.-G., 2015, Phys. Rev. C, 91, 025801

Shapiro S. L., Teukolsky S. A., 1983, Black holes, white dwarfs, and neutron stars: The physics of compact objects. WileyInterscience, New York

Sharma B. K., Centelles M., Viñas X., Baldo M., Burgio G. F., 2015, Astron. Astrophys., 584, A103

Shternin P. S., 2008, Journal of Physics A Mathematical General, 41, 205501

Shternin P. S., Yakovlev D. G., 2008, Physical Review D, 78, 063006

Watanabe G., Iida K., 2003, Phys. Rev. C, 68, 045801

Watanabe G., Iida K., Sato K., 2000, Nuclear Physics A, 676, 455

Watanabe G., Sato K., Yasuoka K., Ebisuzaki T., 2002, Phys. Rev. C, 66, 012801

Williams R. D., Koonin S. E., 1985, Nuclear Physics A, 435, 844

Yakovlev D. G., 2015, MNRAS, 453, 581

Yakovlev D. G., Kaminker A. D., Gnedin O. Y., Haensel P., 2001, Phys. Rep., 354, 1 